\title{
A Giant Dumbbell Shaped Vesico-Prostatic Urethral Calculi
}

\author{
Dr.Adarsh kr chauhan, Dr.Manu Gupta,Dr.A.K Mongia, Dr.Piyush Gupta, \\ Dr.Amit Bhatnagar \\ General Surgery Department Of Subharti Medical College Meerut U.P India
}

\begin{abstract}
Giant vesico-prostatic urethral Calculi is uncommon. The decision about treatment strategy of calculi depends upon the size, shape, and position of the calculus and the status of the urethra. If the stone is large and immovable, it may be extracted via the perineal or the suprapubic approach. In most of the previous reported cases, giant calculi were extracted via the transvesical approach and external urethrotomy. A 60-yearold male patient presented with complaints of lower urinary tract symptoms. Further investigations showed a giant vesico-prostatic urethral calculus secondary to stricture of bulbo-membranous part of the urethra. Surgical removal of calculus was done via transvesical approach. Two calculi were found and extracted. One was a huge dumbbell calculus and the other was a smaller round calculus. This case was reported because of the rare size and the dumbbell shape of the stone. Giant urethral calculi are better managed by open surgery.
\end{abstract}

\section{Introduction}

Urinary calculi are the third most common affliction of the urinary tract, exceeded only by urinary tract infection and pathologic condition of the prostate ${ }^{[1]}$. They are typically asymptomatic and may be associated with benign prostatic hyperplasia, and prostatic cancer ${ }^{[2]}$. Primary prostatic urethral calculi are usually associated with urethral strictures, posterior urethral valve or diverticula. Acute urinary retention might result secondary to a large urethral calculus ${ }^{[3]}$. Giant calculi occurring in prostatic urethra are extremely rare. Only few cases of giant prostatic urethral calculi are reported in the literature. Here we present a case of giant shape calculus in prostatic urethra.

\section{Case Report}

A 60 year-old gentleman, farmer by occupation presented with chief complaints of thinning of urinary stream, straining during micturition, dysuria and feeling of incomplete emptying of bladder for last 10 months. Patient had past history of road traffic accident 2 years ago for which patient had undergone abdominal operation with suprapubic catheterisation and some endoscopic urethral procedure in some other hospital $\mathrm{He}$ was not on proper follow-up after the operation. The patient was unable to state the pathology and operation performed following road traffic accident. Two months after the operation the supra pubic catheter was removed with much difficulty and patient was told that the catheter had broken and some part of it was retained in the bladder. Clinical examination of abdomen revealed a transverse scar in the suprapubic region.

Calculus was palpable in the perianal region and also on per rectal examination. External genitalia were normal.

\section{Investigation}

Haematology: complete blood count, renal function test, liver function test, serum electrolyte, serum calcium levels were within normal limits.

Urinalysis shows pyuria and microscopic haematuria. Urine culture confirmed Escherichia coli colonization. Subsequent imaging with plan X-ray KUB was repeated here which showed a huge calculus which was Dumbbell shaped in the region of bladder and prostatic urethra(figure 1). Computerized tomography (CT) scan displayed a large hyper dense calcified focus of size $4.36 \times 3.65 \mathrm{~cm}$ seen in the urinary bladder and prostatic urethra (figure 2). 


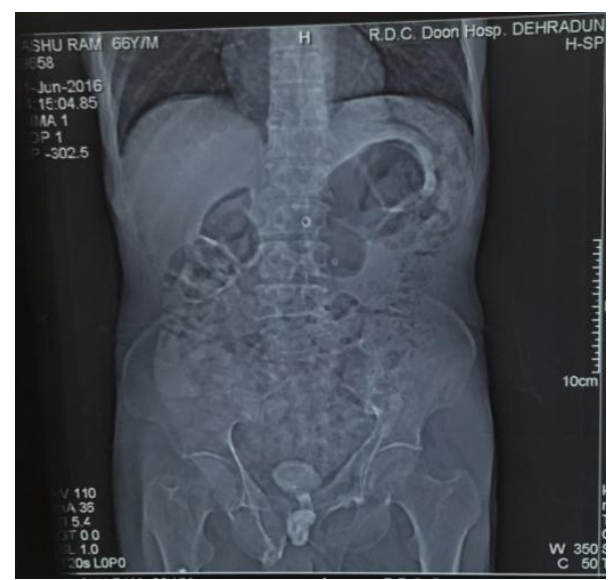

Figure 1. Erect X-ray KUB showing giant calculus in the lower
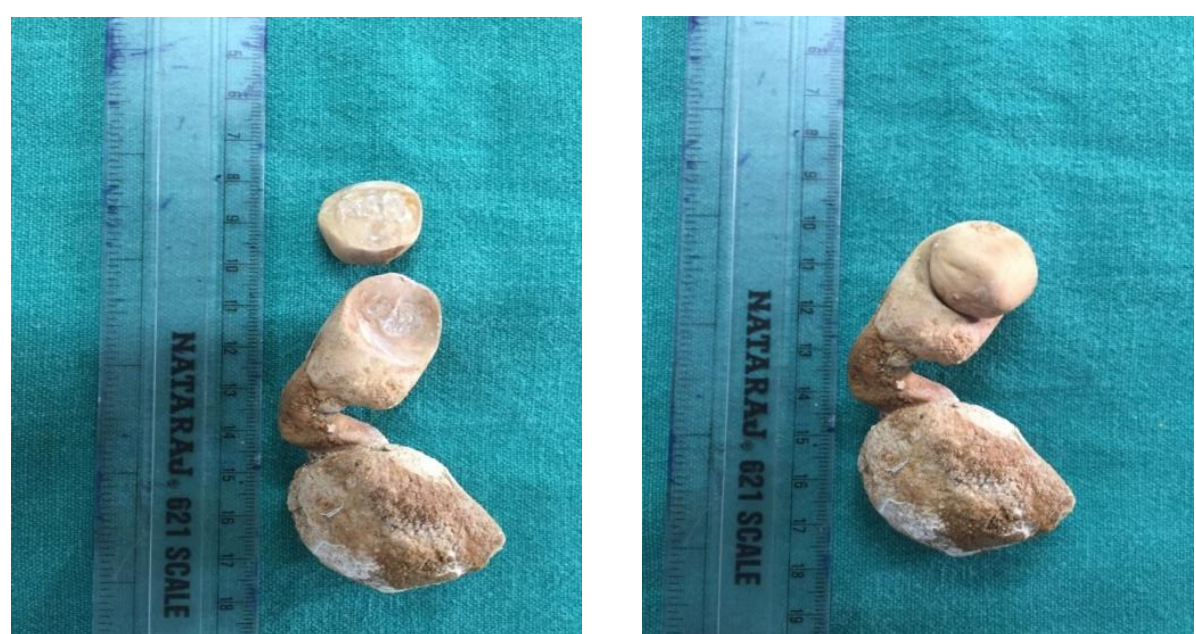

Figure 3. Picture showing two calculi one huge dumbbell shaped calculus and a smaller globular calculus.

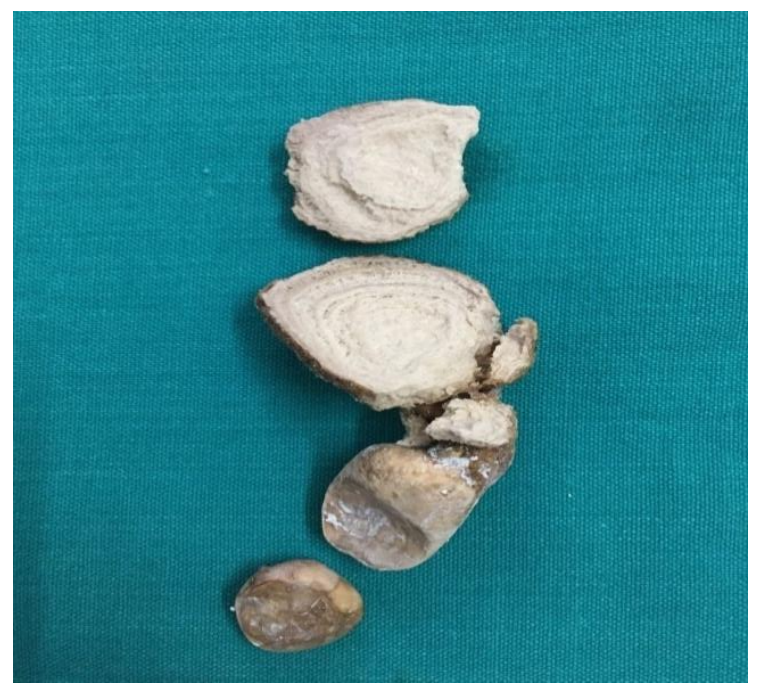

Figure 3. Picture showing cut section of two calculi one huge dumbbell shaped calculus and a smaller globular

\section{Treatment} calculus .

The patient was subsequently admitted to our department. Two days later he underwent for surgical exploration (transvesical)with removal of calculus with bladder neck incision, and visual internal urethrotomy. Two calculi were found and removed. One was a huge dumbbell shape calculus of size around $8.5 \times 3.5 \times 2.5$ $\mathrm{cm}$ and another was a small round shape calculi of size $2 \times 0.5 \mathrm{~cm}$ (Figure 3) The calculus on biochemical examination shows calcium magnesium ammonium phosphate (triple phosphate). Urinary drainage was 
achieved through suprapubic catheter (SPC) and a pelvic drain was also placed. A 16 Fr catheter was passed per urethra. Drain was removed on $5^{\mathrm{TH}}$ postoperative day. Catheter was removed on postoperative day $7^{\mathrm{TH}}$. Patient started to pass urine satisfactorily and SPC was removed. The patient had good urinary stream and no retrograde ejaculation and was subsequently discharge.

\section{Discussion}

Urethral calculi are generally classified as native (those formed de novo in the urethra) or migratory (those formed in the bladder or kidney with secondary descent). Young divides the primary urethral calculi into four groups

Group I: prostatic urethral calculi associated with prostatitis.

Group II: Calculi associated with hypertrophy of the gland.

Group III: Calculi that stimulate carcinoma.

Group IV: Calculi in both the prostatic urethra and the urinary tract.

Swift Joly has classified stones lodged in the posterior urethra into three different categories .

(a) Vesico-Urethral Stones. These stones lie partly in the posterior urethra and partly in the bladder, and therefore lie astride the internal sphincter which is responsible for a constriction, clearly visible on the calculus.

(b) Urethral Stones. Stones are localized to the urethra.

(c) Urethro-Prostatic Stones. These stones lie partly in the pre-formed cavity in the prostate gland.

In our case, It is a Jolly type "a" with both bladder and prostatic urethral component resulting in a dumbbell shape and Young group IV. De novo urethral stone are generally composed of magnesium ammonium phosphate (struvite). In contrast, migratory stones are often composed of calcium phosphate or calcium oxalate. The composition of the stone in our patient is magnesium ammonium phosphate. The possible etiological factors can be urethral stricture, stasis, or stagnation, with urinary infection, foreign bodies, debris, bladder neck obstruction, idiopathic factors, lithogenic diathesis, and schistisomiasis. ${ }^{[7]}$ Less than 20 cases of giant prostatic urethral calculi have been reported in the English literature. They occur more frequently in younger men, unlike microscopic prostatic calculi, which are usually seen in men older than 50 years of age ${ }^{[8,9]}$ This is the first case in our center and probably the largest giant giant dumbbell shape prostatic urethral calculus in English literature. The main symptoms were acute urinary retention, frequency, burning sensation in the urethra on urination, burning sensation in the perineum and/or rectum, or stinging in the anus. Other less frequent symptoms were hematuria, dribbling or incontinence, interruption of the urinary stream, and a history of having passed a stone. ${ }^{[10]}$ Potential complications included bleeding, incontinence, impotence, and stricture formation ${ }^{[3]}$ This patient presented with lower urinary tract symptoms, which cumulated in acute urinary retention.Treatment was influenced by the size, shape, and the position of the calculus and status of the urethra. The stone in this patient was treated by open transvesical prostatolithotomy, bladder neck incision, and bladder neck reconstruction. The patient had good urinary stream and no retrograde ejaculation. Other methods of management of the giant prostatic calculus included radical prostatectomy, open retro pubicprostatolithotomy and endoscopic lithotripsy ${ }^{[10]}$

This case was reported because of the size and the collar-stud nature of the stone. Giant urethral calculi should be treated with open surgery.

\section{References}

[1]. M. L. Stoller, "Urinary stone disease," in Smith's General Urology, E. A. Tanagho and J. W. McAninch, Eds., p. 246, McGraw Hill Lange, 17th edition, 2008.

[2]. Klimas R, Bennett B, Gardner WA., Jr Prostatic calculi: A review. Prostate. 1985;7:91-6.

[3]. Shanmugam TV, Dhanapal V, Rajaraman T, Chandrasekar CP, Balashanmugam KP. Giant urethral calculi.Hosp Med. 2000;61:582.

[4]. Young H. Prostatic urethral calculi. J Urol. 1934;32:660.

[5]. J. C. Barrett, "Giant prostatic calculi," British Journal of Surgery, vol. 45, pp. 267-269, 1957.

[6]. Usta MF, Baykara M, Erdoğru T, Köksal IT. Idiopathic prostatic giant calculi in a young male patient.IntUrolNephrol. 2005;37:295-7.

[7]. Larkin GL, Weber JE. Giant urethral calculus: A rare cause of acute urinary retention. J Emerg Med. 1996;14:707-9.

[8]. Gawande AS, Kamat MH, Seebode JJ. Giant prostatic calculi.Urology. 1974;4:319-21.

[9]. Bedir S, Kilciler M, Akay O, Erdemir F, Avci A, Ozgök Y. Endoscopic treatment of multiple prostatic calculi causing urinary retention. Int J Urol. 2005;12:693-5.

[10]. 10. Virgili G, Forte F, Sansalone S, Attisani F, De Carolis A, Di Stasi SM, et al. Radical prostatectomy as unique chance for huge prostatic stones. Arch ItalUrolAndrol. 2004;76:171-2. 